\title{
BMJ Open Protocol for the residents in action pilot cluster randomised controlled trial (RiAT): evaluating a behaviour change intervention to promote walking, reduce sitting and improve mental health in physically inactive older adults in retirement villages
}

\author{
Cecilie Thøgersen-Ntoumani, ${ }^{1}$ Ashlene Wright, ${ }^{1}$ Eleanor Quested, ${ }^{1}$ Elissa Burton, ${ }^{2}$ \\ Keith D Hill, ${ }^{2}$ Ester Cerin, ${ }^{3,4}$ Stuart J H Biddle, ${ }^{5,6}$ Nikos Ntoumanis ${ }^{1}$
}

To cite: Thøgersen-Ntoumani C Wright A, Quested E, et al. Protocol for the residents in action pilot cluster randomised controlled trial (RiAT): evaluating a behaviour change intervention to promote walking, reduce sitting and improve mental health in physically inactive older adults in retirement villages. BMJ Open 2017;7:e015543. doi:10.1136/ bmjopen-2016-015543

- Prepublication history for this paper is available online. To view these files please visit the journal online (http://dx.doi. org/10.1136/bmjopen-2016015543).

Received 14 December 2016 Revised 27 April 2017 Accepted 11 May 2017

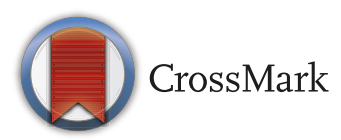

For numbered affiliations see end of article.

Correspondence to

Dr Cecilie Thøgersen-Ntoumani; c.thogersen@curtin.edu.au

\section{ABSTRACT}

Introduction Ageing is accompanied by increased risks of chronic disease, declined functioning and increased dependency. Physical activity is critical to retaining health and independence, but the majority of older people are insufficiently physically active to achieve these benefits and have high levels of sedentary (sitting) time. Activity programmes are often offered in retirement villages; however, their uptake is limited. Furthermore, although the physical environment in and around these villages can play an important role in decisions to be physically active, its role is often overlooked by research in these settings. We aim to develop, implement and evaluate a proof-ofconcept motivationally embellished intervention designed to increase walking, reduce sitting and improve mental health in residents in retirement villages.

Methods and analysis This will be a 16-week pilot intervention using a cluster randomised design with retirement villages as the unit of randomisation and residents as the unit of assessment. Fourteen retirement villages around Perth, Western Australia, will be recruited for the intervention. Objective audits of neighbourhood environments around each village will be completed using the Pathway Environmental Audit Tool. Seven villages will be randomised to the experimental arm and seven to the control arm. Only participants in the experimental arm will receive motivational training. All outcomes will be assessed at baseline, end of intervention and 6-month follow-up. Changes in physical activity levels, sitting time and mental health will be examined. Multilevel modelling will be used to analyse the data. A mixed methods process evaluation will also be conducted.

Ethics and dissemination Ethics approval was granted by Curtin University's Human Research Ethics Committee (HREC2016-0187). The results of the study will be disseminated via peer-reviewed publications, conference presentations and reports to, and seminars with, stakeholders.
Strengths and limitations of this study

- An objective measurement of walking will be used

- The moderating influence of objectively assessed neighbourhood environment characteristics as well as retirement village characteristics will be examined.

- Motivational mediators will be examined to elucidate mechanisms of change.

- Only a 6-month (not 12-month) follow-up will be possible due to resource constraints.

Trial registration The trial is registered with the Australian New Zealand Clinical Trials Registry: ACTRN12616001177448.

\section{INTRODUCTION}

Older adults represent an increasing proportion of the world's population. It is estimated that, by 2050 , adults aged 65 years and above will constitute $16 \%$ of the global population. ${ }^{1}$ The risks of a range of chronic diseases and dependency increase with age, with potentially far-reaching individual and societal costs. However, these risks can be modified through engagement in regular and sustained physical activity. For example, physical activity participation reduces the risks of losing independence, acquiring chronic diseases, including dementia, can increase life expectancy and improve quality of life..$^{2-5}$ However, as people age, physical activity tends to decline, with the majority of older adults not engaging in sufficient amounts of physical activity to maintain or improve 
health. ${ }^{6-9}$ Current guidelines of physical activity for older adults suggest they should engage in at least $150 \mathrm{~min}$ of moderate intensity physical activity per week in bouts of at least $10 \mathrm{~min}$ each, or $75 \mathrm{~min}$ of vigorous intensity activity, or an equivalent combination of the two, per week, in addition to balance (three times per week) and muscle strengthening (twice weekly) activities. ${ }^{10}$

\section{Retirement villages as an opportune setting for the promotion of physical activity}

Retirement villages are highly relevant settings to promote physical activity, as they aim to foster relative independence of residents by encouraging the preservation of functional capacity. It is expected that the number of retirement villages will continue to increase, given the rapidly growing ageing population. Given that retirement villages cater for a relatively broad group of older adults, they represent an important setting in which to examine the efficacy and effectiveness of health behaviour change interventions. Retirement villages differ substantially with regard to the socioeconomic status of their residents. For example, in some retirement villages accommodation is funded by residents themselves, but in other villages residents lease their accommodation or their housing is subsidised. Residents in subsidised tenancy tend to be the most disadvantaged in terms of socioeconomic status, mental health, substance abuse and general health. Indeed, it is well established that socioeconomically disadvantaged populations tend to suffer from more health problems and engage in lower levels of physical activity. ${ }^{11} 12$ Thus, it is critical to examine whether a health behaviour change intervention is equally effective in retirement villages with different socioeconomic groups of residents.

Similar to community-dwelling older adults, residents in retirement villages have opportunities to be physically active. For example, structured exercise programmes and facilities such as swimming pools and gyms are often available in these villages. However, existing programmes and facilities do not appeal to a large proportion of residents. ${ }^{13}$ This observation is consistent with findings showing that only $27 \%$ of older adults in retirement villages in Perth, Western Australia, were sufficiently physically active to accrue health benefits. ${ }^{14}$

\section{Walking as a suitable aerobic activity for older adults}

Walking is a safe and free mode of physical activity that can be self-sustained and that does not require any specialist equipment or skills. ${ }^{15}$ It is also the physical activity of choice for older adults, particularly those who are physically inactive. ${ }^{16} \mathrm{~A}$ systematic review of interventions focused on the promotion of walking showed that walking can be increased by up to $60 \mathrm{~min}$ per week in the general population, at least in the short term. ${ }^{18}$ Furthermore, other evidence suggests that for older adults living with some chronic illness or disability (which does not prevent them from walking), accumulating 5000 steps per day is a realistic goal and leads to clear health benefits. ${ }^{19}{ }^{20}$ Group-based formats, in particular, appear to be very effective in increasing walking in physically inactive individuals, and they are instrumental during the adoption stages of physical activity change. ${ }^{21}$ Indeed, retirement village residents who were interviewed on their perceptions of peer-led group-based walks, walking was labelled as extremely important for socialising with other residents while engaging in physical activities. ${ }^{13}$ These findings are consistent with research conducted with other populations of older adults. ${ }^{22} 23$ The proposed group-based intervention format was also seen by the interviewees in the study by Thøgersen-Ntoumani $e t$ al as a potentially effective means of establishing a healthy lifestyle 'routine', which many new residents found difficult in the transition from home-based living to retirement village living. ${ }^{13}$

\section{Targeting both physical activity and sedentary time}

There is now wide recognition that in addition to increasing physical activity, it is also important to reduce sedentary behaviours ('sitting time'). A meta-analysis has shown that those older people in the highest sitting category, when compared with those in the lowest, have significantly elevated risk for all-cause mortality and chronic diseases such as diabetes, independent of levels of physical activity. ${ }^{24}$ In another meta-analysis, with more than 1 million people, it was shown that watching three or more hours of television per day increased the risk of mortality, after adjusting for levels of physical activity. However, while this was true for most people, those with high levels of physical activity showed no such association between television viewing and risk for mortality. ${ }^{26}$ These findings imply that physical activity and sedentary time need to be directly and independently addressed and that the deleterious effects of high levels of sitting are most pronounced for those who are relatively inactive. Moreover, as moderate-to-vigorous physical activity and sedentary behaviour are largely independent, it is also clear that changes in one behaviour do not necessarily result in changes in the other. Identifying effective strategies to reduce sitting is particularly important in older adults who tend to spend more than 8 hours per day sitting, which is higher than any other age group. ${ }^{27}$

Intervention research directly targeting sedentary behaviour with older adults in particular is, however, sparse. ${ }^{28}{ }^{29}$ In one habit-based sedentary behaviour reduction intervention, the researchers designed a booklet consisting of 16 tips on how to reduce sitting behaviours. ${ }^{30}$ The selection of techniques was guided by Lally and Gardner's habit formation framework and encompassed techniques designed to increase motivation to reduce sedentary behaviour (and increase physical activity), facilitate the translation of intentions into actions and disrupt sitting. Overall, the techniques were received favourably by older sedentary adults. ${ }^{29} \mathrm{~A}$ recent review of studies with adults conducted by Gardner and colleagues suggested that self-monitoring, problem solving, modifying the social and physical environments and providing 
information on the health consequences of sitting were promising behaviour change techniques in interventions designed to reduce sedentary behaviour. ${ }^{28}$

\section{The physical environment}

In line with ecological models of behaviour change, interventions should consider the physical environment alongside individual and social factors. ${ }^{31}$ However, little is known about how the environmental characteristics of one's place of residence impact on the effectiveness of behavioural interventions aimed at increasing walking and reducing sedentary behaviour. ${ }^{32}$ It is plausible to assume that the short and, especially, long-term effects of interventions focused on walking would be partly determined by neighbourhood 'walkability. ${ }^{33}$ This is especially the case for older adults who are likely to be highly influenced by the physical environment, since mobility and independence can be greatly limited by a poorly designed community space. ${ }^{34}$ Specifically, positive associations have been reported between perceived and/ or objectively measured street connectivity, number of walking paths, traffic and crime safety, aesthetics, access to services, residential density and walking for different purposes in older people. ${ }^{3435}$ A recent review of the literature found poor agreement between the perceived and objectively measured neighbourhood environments; each predicted unique variance in physical activity. ${ }^{36}$ Additionally, perceived environmental attributes have been shown to moderate the effectiveness of a motivational intervention focused on walking in middle-aged adults, whereby motivational aids were able to overcome the negative effects of low neighbourhood aesthetic appeal. ${ }^{32}$ Thus, in order to better understand the effectiveness of interventions, it is important to evaluate the moderating effects of both the objectively measured and perceived neighbourhood physical environments. A study with older adults from retirement communities showed that a 3-month intervention focused on addressing individual, social and built environment factors was successful in helping older adults change their perceptions of the environment so participants began to perceive fewer environmental barriers to walking and became more satisfied with their walking environment. ${ }^{37}$ Therefore, it is likely to be important to raise awareness of opportunities to walk in the local environment when implementing walking interventions with retirement village residents.

\section{Self-determination theory (SDT)}

Grounding interventions on sound theory is informative because theory helps facilitate an understanding of why and how intervention strategies work. ${ }^{38}$ One theoretical framework that has shown good potential to promote health behaviour change is SDT. ${ }^{39}$ Although many interventions have shown promise in increasing physical activity levels, a review of 57 interventions with older adults showed short-term but no long-term positive effects. ${ }^{40} \mathrm{SDT}$ identifies enabling factors for long-term adherence to physical activity. ${ }^{41}$ The theory proposes that only autonomous motivation, that is motivation emanating from within the individual, is likely to contribute to sustained behaviour change, especially for behaviours that are important for health but that are not necessarily inherently enjoyable. In contrast, motivation resulting from feelings of guilt or pressure (ie, controlled motivation) is unlikely to result in sustained behaviour change. ${ }^{41}$ The social environment plays an important role in helping individuals internalise motivation for engaging in behaviours. Specifically, the provision of autonomy support (eg, by a walk leader), which is characterised by offering choice, meaningful information in a non-judgemental manner, being empathetic and minimising the use of pressure and control, can facilitate internalised motivation. These propositions have been extensively tested and supported in the healthcare (including physical activity) domain. ${ }^{41-46}$ For example, Thøgersen-Ntoumani and colleagues have shown that incorporating SDT-based motivational principles into the design of a 16-week walking intervention was effective in terms of promoting positive changes in physically inactive middle-aged and older University employees. ${ }^{46}$ Specifically, there were increases in walking, other types of physical activity and mental well-being by the end of the intervention, which were sustained at a 4-month follow-up. It was also shown that it was possible to train walk leaders in the provision of autonomy support to participants. ${ }^{46}$ This intervention format can readily be adapted for use with other population groups, after any necessary customisations.

\section{The present intervention}

The intervention described in this paper will differ from previous physical activity and sedentary behaviour interventions with older adults, thereby adding significant (conceptual and applied) value to the existing literature. A 'train-the-trainer' approach will be adopted as an important part of the intervention, which will entail the training of older residents (subsequently referred to as 'resident ambassadors') in using a motivationally supportive communication style. These resident ambassadors will subsequently guide, motivate and support their peers to adopt and sustain lifestyle changes. It is expected that this support will empower their peers to change the quality of their own motivation to walk more and sit less, thus increasing the likelihood of intervention sustainability in the long term. In contrast to previous walk-based interventions, the reduction of sedentary (sitting) behaviours will be targeted in addition to walking. ${ }^{1947}$ Furthermore, a phased approach will be used in the intervention design whereby organised group support will be phased out and will be gradually replaced by support in developing self-regulated and self-organised walks. This transition will allow participants to take increasing ownership of their behaviours. Finally, in line with recommendations to examine moderators and mediators of health behaviour change, a range of personal, physical environment and village characteristics will be assessed. ${ }^{48}$ 


\section{Aim and study objectives}

The overall aim of the study is to examine the feasibility and preliminary effects of a 16-week motivationally embellished peer-led walking intervention on walking, sitting and mental health in physically inactive residents in retirement villages in Western Australia.

The specific objectives are:

- to assess the feasibility of the intervention with regard to uptake (numbers approached vs numbers consenting to participate), adherence and attrition, as well as overall programme acceptability to resident ambassadors and participants;

- to test the preliminary effects of the intervention on changes in step counts, self-reported sitting and mental health from baseline to the end of the intervention as well as at 6-month follow-up;

- to conduct a process evaluation to (A) assess the degree to which the intervention can be implemented as planned and (B) identify barriers and facilitators to implementation from the perspectives of resident ambassadors and participants;

- to explore the moderating influence of (A) the neighbourhood environment (assessed objectively and via self-reports) and (B) types of tenancy (resident funded, leased and subsidised) on intervention effectiveness;

- to examine motivational mediational mechanisms of behaviour change.

\section{METHODS AND ANALYSES}

\section{Study design}

This will be a 16-week pilot intervention using a cluster randomised design. Data collection will start in March 2017, and it is expected that all assessments will be completed a year later. Retirement villages will be the unit of randomisation, and residents will be the unit of assessment. Participants will be randomly assigned to either control or experimental arm with a 1:1 allocation, stratified by village. A researcher not associated with recruitment or data collection, and who will be blind to village identity, will carry out randomisation via computer software, after recruitment. Allocation concealment will be ensured, as randomisation will not be disclosed until the intervention starts. Fourteen retirement villages in Western Australia will be recruited. Seven villages will be randomised to the experimental arm and seven to the control arm. This number of villages has been selected because 30 villages would be considered the minimal sample size to estimate environmental moderating effects in a definitive randomised controlled trial (RCT). ${ }^{49}$ Targeting approximately half of the minimal sample size of villages will provide for a sufficiently robust estimate to inform power analyses for a future definitive trial. We aim to recruit villages (and match them as much as possible across both arms) that will be characterised by varying levels of social disadvantage (lease-for-life, resident funded or subsidised tenancy), physical environment characteristics (eg, perceived safety, safety from traffic and proximity to amenities) and levels of deprivation (as defined through Socio-Economic Indexes for Areas scores). We will recruit an average of 15 resident participants (total $\mathrm{n}=210$ ) and five resident ambassadors (total $n=70$ ) from each village. However, given the pragmatic nature of the intervention and the fact that the sizes of villages differ greatly, the numbers of ambassadors and walkers are likely to vary per village. All participants, village coordinators and the data analyst will be blinded to allocation, given that in both arms participants and resident ambassadors will receive training without knowing that the content of the training will differ across villages. There will be no circumstances under which unblinding for those individuals will be necessary. Research assistants (RAs) who will collect data will be unblinded due to practical constraints.

The protocol has been prepared in accordance with guidelines by the Standard Protocol Items: Recommendations for Interventional Trials and Template for Intervention Description and Replication. ${ }^{5051}$ Completed checklists are provided as additional files.

\section{Recruitment, participants, eligibility and screening}

All recruitment will be done by the research team that will liaise with providers of retirement villages in Perth, Western Australia, and nearby areas. When support in principle is obtained from the providers, the research team will make contact with the village coordinators and, following approval for recruitment, they will arrange suitable methods and opportunities for the research team to promote the intervention among the residents of the villages. Forms of recruitment will be face-to-face contact (eg, talks introducing the project as part of coffee mornings), and via posters, flyers and letters posted in residents' letter boxes. Resident ambassadors, who will volunteer for the role, will be permanent residents in the retirement villages, aged 60 years or above, who are able to communicate well in English, can provide consent, are already physically active (ie, meet the current Australian Government recommendations of physical activity for health) and have a willingness and commitment to take on the ambassador role. ${ }^{52}$ Walk group participants will be permanent village residents, aged 60 years or above, who are able to communicate well in English, provide consent, participate in baseline assessments, have no terminal illness or health problems that prevent them from walking (we will include those who use walking sticks and walking frames), do not have a known dementia diagnosis, can walk continuously on a flat surface at a light/moderate pace for $15 \mathrm{~min}$, have had fewer than two falls in the past 3 months and do not currently meet the specific Australian Government guideline for older adults to accumulate at least $30 \mathrm{~min}$ of moderate intensity physical activity on most days of the week (ie, recommendation 3 of 'Choose Health, Be Active'). ${ }^{52}$ Screening of ambassadors and group walk participants' physical activity levels will be done using the two-stage approach outlined by Vandelanotte $e t a l .^{53}$ Participants will also be asked to complete 
the Physical Activity Readiness Questionnaire to ensure that there are no contraindications for walking. ${ }^{54}$

\section{Intervention}

The role of resident ambassadors

The role of the resident ambassadors will be to help promote the increase of walking and the reduction of sitting behaviours in the resident participants. The ambassadors will be trained to implement specific strategies to help resident participants achieve this. Each ambassador will be matched up with 3-5 residents whom they will work with for the duration of the intervention. The decision to have three to five residents per group was informed by preparatory work conducted by the research team. ${ }^{13}$ The ambassadors will provide a range of different types of support, including leading weekly small group walks, and encouraging participants to engage in self-organised walks. Their involvement will be phased out after week 10 of the intervention.

\section{Training of resident ambassadors}

To prepare the ambassadors for their role, initially all resident ambassadors will participate in a practical groupbased 2-hour workshop, which will be led by members of the research team. In the control arm, the workshop will consist of information on the benefits of increased walking and reduced sitting, training in how to lead group walks in a safe manner, what to do in case of emergencies and the tasks needed to coordinate and lead the group walks. In the experimental arm, the resident ambassadors will receive similar information (some of it will be in handout format due to time constraints), but they will also receive training on the major types of motivation proposed by SDT, and they will be asked to reflect on their own motivation for their role. Resident ambassadors in both arms will receive printed material to support learning.

In the experimental arm, resident ambassadors will also receive an additional 2-hour workshop later in the same day, delivered by members of the research team. In this workshop, the ambassadors will be introduced to the basic principles of the motivational framework underpinning the intervention (ie, SDT) and will engage in practical exercises that will aim to show how to support other residents' autonomous motivation for walking and for reducing sitting behaviours. Specifically, the different motivations underpinning engagement in these behaviours will be described and illustrated. The resident ambassadors will then be introduced to ways in which autonomous motivation can be supported. For example, the resident ambassadors will be trained to encourage participants' involvement and opinions in making choices (eg, walking routes and ways to break up sitting time), use open-ended questions, be empathetic, acknowledge difficulties or negative emotions, develop feelings of belongingness, provide competence-building feedback, avoid judgemental or controlling language, provide rationales for activities and encourage personal responsibility and initiative. The resident ambassadors will be encouraged to identify concrete examples of how they can put these principles into practice. They will also be provided with relevant examples. Two weeks into the intervention, a third 2-hour workshop will be offered to the ambassadors in the experimental arm to share successes and challenges in implementing the motivational strategies taught. Brainstorming will be encouraged, and solutions will be identified. In between the second and the third workshops, as well as after the third workshop, resident ambassadors in the experimental arm will use training material to achieve weekly goals (eg, helping participants feel successful) via the implementation of SDT-based motivational strategies. The resident ambassadors in the control group will not receive the motivational training, but the motivational training will be made available to them at the end of the project. Due to geographical distance between the villages in the same conditions, all workshops will be delivered in the ambassadors' own retirement villages.

\section{Group sessions for residents}

In both arms, the residents will receive at the beginning of the intervention one group-taught 2-hour session, delivered by the research team, on the benefits of walking and reducing sitting time for health. In this session, they will also be provided with outdoor walk route maps and information on step counts for specific walks to destinations inside and outside their villages. The participants in the experimental arm will receive the same information but in a more condensed form during the workshop (but with more detailed handouts to read afterwards). They will also be offered training on motivation using SDT principles. Given that SDT argues that facilitating the understanding of the importance of the activity will help individuals to self-endorse it, the participants in the experimental arm will undertake exercises in which they will reflect on how and in what ways the programme will be beneficial to them and how it aligns with their personal values. Furthermore, the participants will receive training in behaviour change techniques (eg, implementation intentions, self-monitoring and goal setting) in an effort to optimise and support motivation. For example, participants will be asked to reflect on personal barriers to walking and reducing sitting time and how to overcome them by planning a response for anticipated obstacles (ie, 'implementation intentions'). They will be given self-monitoring exercises (in addition to logging step counts) so that they can become more aware of how their walking and sitting behaviour patterns change and how to cope with tempting situations (eg, sedentary alternatives) that might prevent them from undertaking their walks or breaking up their sitting time. Goal setting principles will be illustrated, and relapse prevention will be discussed with suggestions of how to get back on track following temporary setbacks. Furthermore, they will also be taught behaviour change techniques regarding ways of breaking up long periods of sitting. A tips sheet outlining specific strategies will be produced based on the results 
of a review by Gardner and colleagues and preliminary qualitative work which is currently being conducted by our team with 20 residents. ${ }^{28}$ As for the resident ambassadors, the workshops will be delivered in the residents' own retirement villages.

Participants in both arms will be provided with log-books for the documentation of step counts. They will also be encouraged to use a tick sheet to indicate how many times per day they took a break from sitting (when sitting more than $30 \mathrm{~min}$ at a time). ${ }^{55}$

The participants in the experimental arm will be given motivational booklets that will include week-byweek guidance on implementing the behaviour change techniques covered in the workshop. They will also be supported in successfully using these techniques by the researchers by biweekly visits (either visiting participants individually or in groups) or phone calls (which will also aim to facilitate participant retention to the study and to collect information on any adverse events). These visits and phone calls will be weaned off gradually during the 16-week intervention to prevent participants becoming reliant on this support. Participants in the control group will not be given motivational booklets but will receive a general fact sheet with practical examples of increasing physical activity and reducing sedentary behaviour. The full intervention package will be offered to the villages in the control arm at the end of the project. The intervention material will be made available to participants in the current trial in hard copy and online on completion of the testing of the intervention (eg, via the project website: www.riat.com.au).

\section{Group-led walks}

Group-led walks constitute an important component of the intervention. Twenty-to-thirty minute group-led walks, which will be led by resident ambassadors, will be offered during the mornings and afternoons for the first 10 weeks to participants in both arms. Participants of similar walking capability will be put in the same group based on results of a 6 min walk test. Participants will be encouraged to partake in three group walks per week. Duration and intensity of the walks will be adapted to the needs of the groups. The routes will be risk assessed by the researchers prior to the intervention commencing. Criteria for assessment will include assessing the level of pavements, street lighting, congested traffic areas, traffic lights, marked foot crossings for pedestrians or pedestrian island included in the midpoint of the road. The routes will be planned around the retirement villages to enable those participants who feel unable to walk for the full duration of the walk to walk only part of the route. In addition to the group-led walks, the residents in both conditions will also be encouraged during the first 10 weeks to engage in at least two self-organised walks per week (indoor walk routes will also be planned and promoted). In the final 6 weeks, the participants in both arms will be encouraged to self-organise all their walks in smaller groups or individually.

\section{Residents in action trial (RiAT) intervention procedures}

An outline of the procedures for the trial is presented in figure 1.

\section{Feasibility-related outcomes}

Descriptive data on uptake will be recorded by the research team during the recruitment process. The numbers of participants consenting to participate will be compared with those approached. Attendance at the group walks will be monitored by the resident ambassadors via a group walk register. Participant residents will be asked to document engagement in self-organised walks that are at least $10 \mathrm{~min}$ long in their logbooks. These data will be used to assess level of adherence. Attrition will be assessed by examining dropout rates that will be recorded by the research team (including dates); reasons for dropout will be logged where possible. Furthermore, acceptability of the intervention will be assessed via a nine-item scale adapted from our previous work . Example items include 'I enjoyed the programme' and 'I would recommend this programme to other people'. Response options range from 1 (strongly disagree) to 7 (strongly agree).

\section{Outcome measures}

Primary outcome

The primary outcome will be step counts collected via participants' $\log$ books and will be assessed at baseline, at the end of the intervention and at a 6-month follow-up. For self-monitoring purposes, New Lifestyles NL-1000 pedometers, which have been shown to accurately measure steps at varying speeds, will be provided to participants in both arms 2 weeks prior to the start of the intervention. ${ }^{56}$ Data collected in the first week will not be analysed to account for possible reactivity effects. ${ }^{57}$ The step counts from the second week will be used as an estimate of baseline walking.

\section{Secondary outcomes}

Self-reported physical activity

Validated self-report measures will be used to assess the participants' typical physical activity levels and sedentary behaviours. Habitual physical activity will be measured via the Physical Activity Scale for the Elderly. ${ }^{58}$ This is a 12-item scale that assesses types of physical activities older adults have participated in during the past 7 days (eg, gardening, housework, walking and shopping). This scale has been validated extensively. ${ }^{59}$

\section{Sitting}

Sitting behaviours will be assessed using a questionnaire developed to assess sedentary behaviours in older adults. ${ }^{60}$ The scale assesses the amount of time (hours and minutes) people spend in a range of sedentary behaviours (eg, watching TV, using the computer and reading) over the past week. The scale has been validated with older adults. ${ }^{60}$

General health, functioning and quality of life

The widely used 12 item Short Form Survey (SF-12) will be administered to assess perceptions of general health 
Recruitment

$n=14$ retirement villages $n=70$ resident ambassadors and $n=210$ resident participants

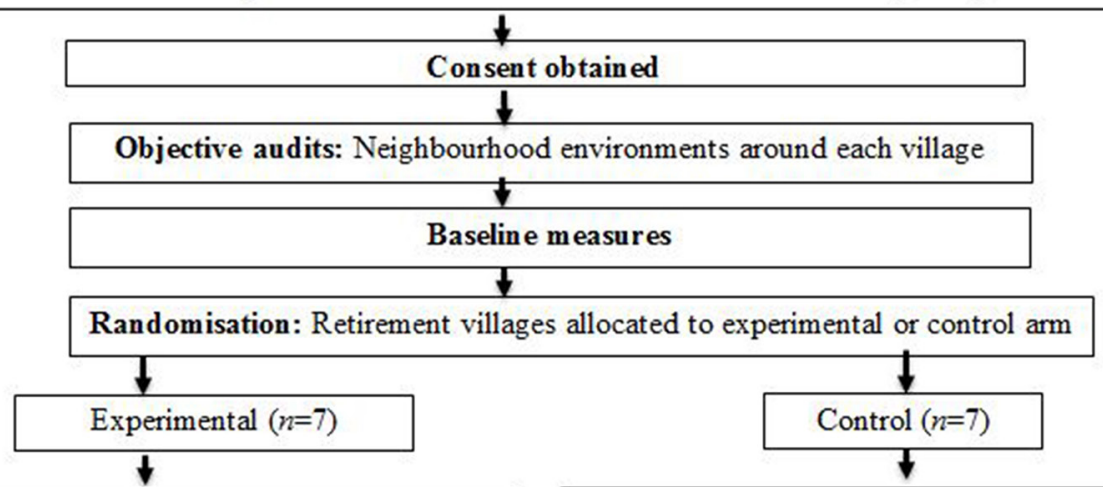

Ambassadors $(n=35)$ : Three workshops on ambassador training and motivational training

Resident participants ( $\mathrm{n}=105)$ : One workshop on benefits of walking and reducing sitting and on motivation and behaviour change strategies. Handout material and log books provided

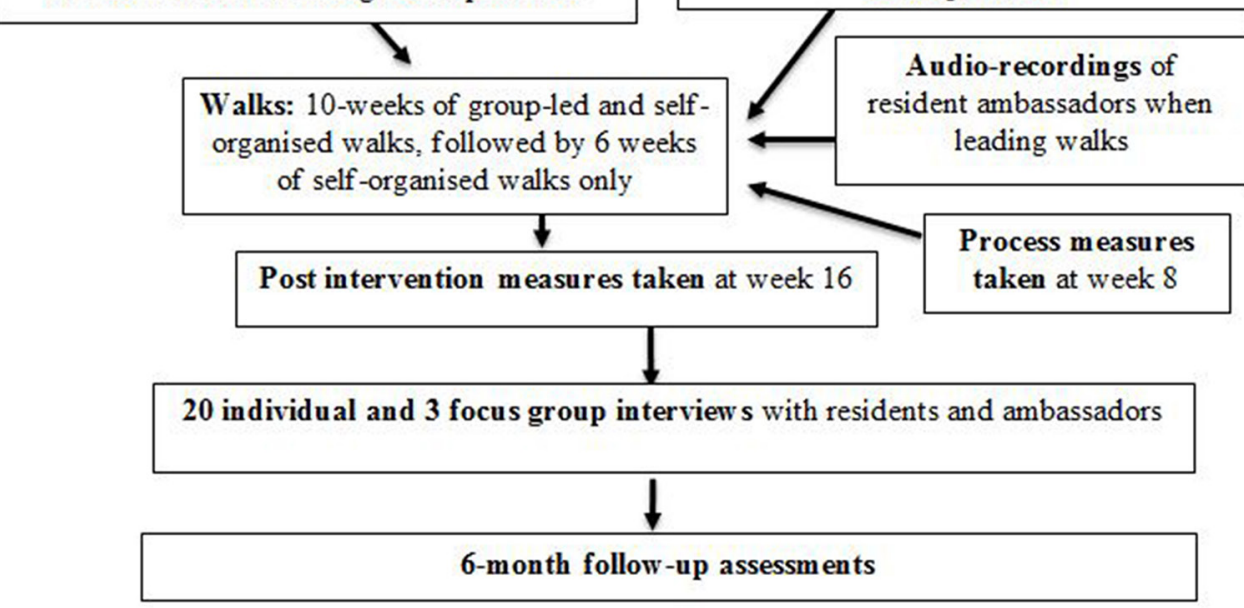

Ambassadors $(n=35)$ : One workshop on ambassador training

Resident participants ( $\mathrm{n}=105)$ : One workshop on benefits of walking and reducing sitting. Handout material and log books provided

Figure 1 Outline of trial procedures.

and functioning. ${ }^{61}$ The scale asks respondents to respond to statements regarding their general physical and mental health over the last 4 weeks (eg, 'During the past 4 weeks, how much did pain interfere with your normal activities?'; 'During the past 4 weeks, did you have a lot of energy?'). Good levels of reliability and validity of the SF-12 have been reported in research with older adult populations. ${ }^{61}$ Furthermore, the Dartmouth COOP Functional Assessment Charts will be used to assess quality of life. ${ }^{62}$ This scale identifies nine domains relevant to quality of life (ie, physical fitness, feelings, daily activities, social activities, pain, change in health, overall health, social support and quality of life), and a reference is made to the past 4 weeks. An example item is 'During the past 4 weeks, how much have you been bothered by emotional problems such as feeling anxious, depressed, irritable or downhearted and sad?' All items are rated on a scale ranging from 1 (not at all) to 5 (extremely). A previous study with a sample including older adults reported test-retest correlation coefficients between 0.93 and $0.99{ }^{63}$

\section{Mental health and well-being}

The Hospital Anxiety and Depression Scale (HADS) will be used to measure anxiety and depressive symptoms. ${ }^{64}$ The scale comprises 14 items: seven items to measure anxiety (eg, 'I can sit at ease and feel relaxed') and seven items to assess depression (eg, 'I still enjoy the things I used to enjoy'). The items are scored on a four-point scale ranging from 0 to 3 . The psychometric properties of the HADS have been widely supported in previous research. ${ }^{65}$ Perceptions of loneliness will be measured using three items psychometrically validated by Hughes and colleagues. ${ }^{66}$ An example item is 'how often do you feel you lack companionship' with responses including 'hardly ever', 'some of the time' and 'often'. Feelings of energy will be assessed using the Subjective Vitality scale, which comprises six items. ${ }^{67}$ One example of the six items is 'I feel alive and vital', with responses ranging from 1 ('not at all true') to 7 ('very true'). Support for the scale's psychometric properties has been reported by Bostic et al.$^{67}$ See table 1 for an overview of all outcome measures. 


\begin{tabular}{|c|c|}
\hline Outcome variables & Measuring instrument \\
\hline \multicolumn{2}{|l|}{ Primary outcome } \\
\hline $\begin{array}{l}\text { Physical activity: } \\
\text { step counts }\end{array}$ & $\begin{array}{l}\text { NL-1000 pedometer with step } \\
\text { counts recorded in a logbook }\end{array}$ \\
\hline \multicolumn{2}{|l|}{ Secondary outcomes } \\
\hline $\begin{array}{l}\text { Self-report physical } \\
\text { activity }\end{array}$ & $\begin{array}{l}\text { The Physical Activity Scale for the } \\
\text { Elderly }\end{array}$ \\
\hline $\begin{array}{l}\text { Self-report sitting } \\
\text { behaviours }\end{array}$ & Gardiner Sedentary Behaviour Scale \\
\hline $\begin{array}{l}\text { General health and } \\
\text { functioning }\end{array}$ & 12 item Short Form Survey \\
\hline Quality of life & $\begin{array}{l}\text { Dartmouth COOP Functional } \\
\text { Assessment Charts }\end{array}$ \\
\hline Mental health & $\begin{array}{l}\text { The Hospital Anxiety and Depression } \\
\text { Scale and Loneliness Scale }\end{array}$ \\
\hline Feelings of energy & Subjective Vitality Scale \\
\hline
\end{tabular}

Note: Assessments for all outcomes will be taken at baseline, end of intervention (16 weeks) and a 6-month follow-up.

\section{Process evaluation measures}

To examine the degree to which the resident ambassadors use the taught strategies as planned, we will audio-record three randomly selected group-led walks for each of the resident ambassadors (the ambassadors will be asked to audio-record every walk they do). A rating sheet will be constructed for the purposes of this study and will be used by two blinded RAs to code the verbal provision of SDT-based motivational strategies. Each transcript will be coded independently by the two coders, and levels of inter-rater agreement will be calculated.

Additionally, in line with methods reported by Matei et al, logbooks and tick sheets will be collected from participants after the intervention to assess the extent to which behaviour change techniques and tips, provided as part of the workshops and the booklets, were used by the participants. ${ }^{30}$ Information on barriers and facilitators of intervention implementation will be collected via 20 individual and 3 focus group interviews (each with 4-6 individuals) after the trial, with residents (high and low adherers) and resident ambassadors in both arms. Reasons for dropout will also be explored for participants who withdraw from the study at any time point, either via a short questionnaire or a brief exit interview.

\section{Moderating variables: characteristics of the neighbourhood environment and type of tenancy}

Characteristics of the neighbourhood environment will be assessed at baseline only using validated self-report and environmental audit measures (Path Environment Audit Tool). ${ }^{68} 69$ The self-report measure will assess perceived pedestrian safety, safety from crime and traffic and neighbourhood aesthetics. The PEAT is an audit tool assessing four domains: design features, amenities, maintenance and pedestrian safety from traffic. ${ }^{69}$ Walking routes within $1 \mathrm{~km}$ from the selected retirement villages will be identified. Two RAs, who will be blinded to the randomisation, will be trained by the research team in using the PEAT manual. Both auditors (RAs) will assess each identified route. Any discrepancies in ratings will be resolved via discussion between the two raters. Scores on each domain of the PEAT will be computed for each walking route. Sites will be classified as being high-walkability or low-walkability on a specific PEAT domain based on median splits. Information on type of tenancy (resident funded, lease-for-life or subsidised) will be provided by the retirement villages.

\section{Motivation-related mediation variables}

Perceptions of autonomy support. Perceptions of autonomy support provided by the resident ambassadors will be measured via the autonomy support scale developed by Williams and colleagues. ${ }^{70}$ An example item is "My resident ambassador encourages me to ask questions...', and items are rated using a seven-level scale ranging from strongly disagree to strongly agree. High internal reliability coefficients have been reported in previous studies. ${ }^{71}$

Motivation for walking will be assessed using the Behavioural Regulation for Walking Questionnaire. ${ }^{72}$ This 23-item scale includes subscales assessing 'motivation' for walking (eg, 'I don't see why I should have to walk'), external regulation ( $\mathrm{eg}$, 'I walk because other people say I should'), introjected regulation (eg, 'I feel guilty when I don't walk'), identified regulation (eg, 'I value the benefits of walking'), integrated regulation (eg, 'I consider walking to be part of my identity') and intrinsic motivation (eg, 'I find walking a pleasurable activity') for walking. The items are measured on a five-point scale ranging from 'not true for me' to 'very true for me'. Evidence for the scale's adequate psychometric properties has been previously reported. $^{72}$

\section{Data management}

All hardcopy data will be stored securely in locked filing cabinets at the host university. These hardcopies will include participant ID only. The document linking participant IDs with any identifying information will be kept securely on a password-protected computer and stored on the university's secure server. Consistent with data management policy, all data will be kept securely for 7 years, after which it will be destroyed. Only the research team will have access to the data.

\section{Analyses}

\section{Quantitative analysis}

The main purpose of the analyses is to develop reliable power estimates for a subsequent definitive trial. Specifically, descriptive statistics on variable means and adherence to, and drop-out from, the intervention will be calculated. Furthermore, internal reliability coefficients for questionnaire data will be estimated. The main analyses will be conducted using multilevel modelling (also called mixed-effects modelling) on a per-protocol and on an intention-to-treat basis, adjusting for baseline scores. 
Multiple imputation will not be used to replace missing values, as multilevel modelling can handle well cases with missing values. ${ }^{49}$ This type of analysis is superior to standard regression analyses and repeated measures analyses of variance as it adjusts standard errors to take into account village clustering effects. ${ }^{49}$ We will compare mean scores and rates of change across arms for walking and sitting behaviours and all other outcomes; we will also examine individual variation from mean trends. Perceived environmental and types of tenancy (lease for life, resident funded and subsidised tenancy) will be analysed as moderators of the effects of arm allocation on the outcomes. Mediation analysis will be conducted via path analysis, adjusting for cluster effects. CIs of outcome variability and effect sizes will be calculated. An upper $90 \%$ confidence limit of the intraclass correlation coefficient of the primary outcome will be estimated to inform the sample size calculation for a future definitive clustered RCT.

\section{Qualitative analysis}

Data will be transcribed verbatim and coded using QSR NVivo. Thematic analysis using a framework approach will be used to analyse the data from the individual and focus group interviews. ${ }^{73}$ Two independent coders will be used in the analysis process, and peer-review procedures, using other members of the research team, will be employed to challenge interpretations of the data. Any disagreements will be followed by a discussion until consensus is reached. Furthermore, member checking will be adopted to enhance trustworthiness.

\section{Sample size calculation}

The study is not formally powered to detect differences in outcomes, but is designed to generate primary outcome data to power a definitive trial. ${ }^{74}$ The size of the pilot will enable estimation of various useful information (recruitment rates, retention rates, questionnaire response rates, effect size and variability) to be calculated with reasonable precision.

\section{ETHICS AND DISSEMINATION}

Ethics approval for the trial has been granted by Curtin University's Human Research Ethics Committee (HREC2016-0187). All ambassadors and resident participants will be screened for eligibility by the research associate when expressing an interest to take part in the project. Following these checks, they will provide written informed consent. It will be made clear to participants that they will be free to withdraw from the programme at any point without giving a reason. Any significant modifications to the protocol of this study will be subjected to university ethics approval and will be subsequently documented in the Australian and New Zealand Clinical Trials Registry.

All ambassadors and resident participants who take part in the trial will receive a written summary of the results at the end of the project. The findings from the study will also be reported to various stakeholder groups via written reports and seminars. These stakeholder groups will include, but may not be limited to, retirement village providers, resident associations, ageing organisations, aged care providers, policy makers and urban planners. The findings will also be disseminated widely via the media offices of the participating universities, partner organisations, via social media channels including Twitter (@ResinAction2016) and the project website (www.riat. com.au) and to other academics and policymakers via peer-reviewed journal articles and conference presentations.

\section{DISCUSSION}

RiAT is a motivationally embellished pilot cluster RCT, which aims to help physically inactive residents of retirement villages to increase levels of walking, reduce sitting,and improve mental health. The intervention will use strategies to specifically target older adults in retirement villages who do not currently achieve the guidelines to improve or maintain health and wellbeing. These groups are in greater need with regard to improving health and well-being and have greater scope for improvement than their more active counterparts. Any potentially positive impacts of the intervention on individuals' health, well-being, independence and functioning could reduce the risk of these residents going into residential care settings (such as nursing homes) or delay such moves. This in turn could have substantial public health implications in terms of improved quality of life and reduced healthcare expenses. As part of the current pilot trial, we will record trial expenses relating to workshop delivery and production of training material that could inform a health economic analysis in a future definitive trial.

In contrast to previous trials, the present intervention uses a peer-led approach whereby resident ambassadors are motivationally trained to support their less physically active peer residents. Indeed, studies employing the theoretical framework underpinning the intervention (ie, SDT) have shown consistently that the provision of need supportive strategies from key agents within the social environment helps individuals facilitate improvements in quality of motivation and leads to sustained behavioural engagement and positive health and wellbeing outcomes. ${ }^{45}$ An important contribution of the study to the extant SDT literature is examining whether the physical environment and types of tenancy moderate intervention effectiveness.

Beyond intervening at the level of the social environment (ie, training ambassadors), the trial also incorporates training to equip residents with skills to self-sustain both physical activity and sedentary behaviour changes over the longer term, using evidence-based behaviour change techniques. This is critical because previous research has shown that when older adults begin activity programmes, participation is often short lived. ${ }^{76}$ A thorough process 
evaluation will enable the identification of techniques that are more versus less effective, which will inform future intervention efforts in this, and similar, settings.

The results of the present pilot RCT will inform the testing of a definitive trial. If that trial is successful, the intervention programme can be packaged and rolled out for use by providers of physical activity programmes for older adults across Australia and other countries if appropriate. Furthermore, the intervention, or adapted versions of it, can be used across different types of accommodation, or complement existing physical activity programmes for older adults. To facilitate its adoption, the intervention material will become freely available in both printed and online formats following the definitive trial.

\section{Author affiliations}

${ }^{1}$ Health Psychology \& Behavioural Medicine Research Group, School of Psychology and Speech Pathology, Curtin University, Perth, Western Australia, Australia ${ }^{2}$ School of Physiotherapy and Exercise Science, Curtin University, Perth, Western Australia, Australia

${ }^{3}$ Institute for Health and Ageing, Australian Catholic University, Melbourne, Victoria, Australia

${ }^{4}$ School of Public Health, The University of Hong Kong, Hong Kong, China ${ }^{5}$ Institute of Sport, Exercise \& Active Living, Victoria University, Melbourne, Victoria, Australia

${ }^{6}$ Institute for Resilient Regions, University of Southern Queensland, Springfield, Queensland, Australia

Correction notice This paper has been amended since it was published Online First. Owing to a scripting error, some of the publisher names in the references were replaced with 'BMJ Publishing Group'. This only affected the full text version, not the PDF. We have since corrected these errors and the correct publishers have been inserted into the references.

Contributors CT-N, EB, KDH, EC, SJHB and NN conceived the project and obtained the project funding. $E Q$ and $N N$ assisted in the development of the intervention materials. CT-N is coordinating the project. AW is managing the project on a dayto-day basis and is leading the data collection and will supervise causal research assistants. CT-N and AW drafted the manuscript, and all authors read, edited and approved the final version of the manuscript.

Funding This work is supported by the Western Australian Health Promotion Foundation (Healthway), grant number: 24258. EC is supported by an Australian Research Council Future Fellowship (FT3 \#140100085).

Competing interests SJHB: funding has been received since 2013 for consultancy work from Fitness First, Nuffield Health and Unilever. None of these are currently active. Funding was received in 2016 for consultancy work for Halpern PR Limited. In-kind support through the provision of a sit-to-stand desk was provided by Ergotron from 2012 to 2014. Advice has been requested by and offered to Active Working, Get Britain Standing and Bluearth, none with funding.

Ethics approval Curtin University's Human Research Ethics Committee.

Provenance and peer review Not commissioned; externally peer reviewed.

Open Access This is an Open Access article distributed in accordance with the Creative Commons Attribution Non Commercial (CC BY-NC 4.0) license, which permits others to distribute, remix, adapt, build upon this work non-commercially, and license their derivative works on different terms, provided the original work is properly cited and the use is non-commercial. See: http://creativecommons.org/ licenses/by-nc/4.0/

(C) Article author(s) (or their employer(s) unless otherwise stated in the text of the article) 2017. All rights reserved. No commercial use is permitted unless otherwise expressly granted.

\section{REFERENCES}

1. World Health Organisation(WHO). Global aging and health. 2016 http://www.who.int/ageing/publications/global_health.pdf (cited 2016 May 28.).

2. Marques EA, Baptista F, Santos DA, et al. Risk for losing physical independence in older adults: the role of sedentary time, light, and moderate to vigorous physical activity. Maturitas 2014;79:91-5.

3. Larson EB, Wang L, Bowen JD, et al. Exercise is associated with reduced risk for incident dementia among persons 65 years of age and older. Ann Intern Med 2006;144:73-81.

4. Singh MA. Exercise to prevent and treat functional disability. Clin Geriatr Med 2002;18:431-62.

5. Rejeski WJ, Mihalko SL. Physical activity and quality of life in older adults. J Gerontol A Biol Sci Med Sci 2001;56 Spec No 2:23-35.

6. Hallal PC, Andersen LB, Bull FC, et al. Lancet Physical Activity Series Working Group. Global physical activity levels: surveillance progress, pitfalls, and prospects. Lancet 2012;380:247-57.

7. Saarloos D. PsPAFPA S, ed. The Baby Boomers and Beyond Report: physical activity levels of older western Australians [Internet]. 2006:5-15. http://eprints.qut.edu.au/67175/1/Saarloos_2008_-_The_ baby_boomers_and_beyond_report.pdf.

8. Tucker JM, Welk GJ, Beyler NK. Physical activity in U.S.: adults compliance with the Physical Activity Guidelines for Americans. Am J Prev Med 2011;40:454-61.

9. Jefferis BJ, Sartini C, Lee IM, et al. Adherence to physical activity guidelines in older adults, using objectively measured physical activity in a population-based study. BMC Public Health 2014;14((1)):382.

10. World Health Organisation. Global recommendations on physical activity for health. WHO 2010.

11. Gidlow $\mathrm{C}$, Johnston LH, Crone D, et al. A systematic review of the relationship between socio-economic position and physical activity. Health Educ J 2006;65:338-67.

12. Cleland CL, Tully MA, Kee F, et al. The effectiveness of physical activity interventions in socio-economically disadvantaged communities: a systematic review. Prev Med 2012;54:371-80.

13. Thøgersen-Ntoumani $\mathrm{C}$, Ntoumanis $\mathrm{N}$, Uren $\mathrm{H}$, et al. Perceptions of group-based walks and strategies to inform the development of an intervention in retirement villages: perspectives of residents and village managers. J Aging Phys Act 2017;25:261-8.

14. Nathan A, Wood L, Giles-Corti B. Examining correlates of selfreported and objectively measured physical activity among retirement village residents. Australas J Ageing 2014;33:250-6.

15. Morris JN, Hardman AE. Walking to health. Sports Med 1997;23:306-32.

16. Siegel PZ, Brackbill RM, Heath GW. The epidemiology of walking for exercise: implications for promoting activity among sedentary groups. Am J Public Health 1995;85:706-10.

17. Merom D, Pye V, Macniven R, et al. Prevalence and correlates of participation in fall prevention exercise/physical activity by older adults. Prev Med 2012;55:613-7.

18. Ogilvie $\mathrm{D}$, Foster $\mathrm{CE}$, Rothnie $\mathrm{H}$, et al. Interventions to promote walking: systematic review. BMJ 2007;334:1204.

19. Mutrie N, Doolin O, Fitzsimons CF, et al. Increasing older adults' walking through primary care: results of a pilot randomized controlled trial. Fam Pract 2012;29:633-42.

20. Tudor-Locke C, Hart TL, Washington TL. Expected values for pedometer-determined physical activity in older populations. Int $\mathrm{J}$ Behav Nutr Phys Act 2009;6:59.

21. Kinnafick FE, Thøgersen-Ntoumani C, Duda JL. Physical activity adoption to adherence, lapse, and dropout: a self-determination theory perspective. Qual Health Res 2014;24:706-18.

22. Gallagher NA, Gretebeck KA, Robinson JC, et al. Neighborhood factors relevant for walking in older, urban, African American adults. $J$ Aging Phys Act 2010;18:99-115.

23. Stathi A, Gilbert H, Fox KR, et al. Determinants of neighborhood activity of adults age 70 and over: a mixed-methods study. J Aging Phys Act 2012;20:148-70.

24. Wilmot EG, Edwardson CL, Achana FA, et al. Sedentary time in adults and the association with diabetes, cardiovascular disease and death: systematic review and meta-analysis. Diabetologia 2012;55:2895-905.

25. de Rezende LF, Rey-López JP, Matsudo VK, et al. Sedentary behavior and health outcomes among older adults: a systematic review. BMC Public Health 2014;14:333.

26. Ekelund U, Steene-Johannessen J, Brown WJ, et al. Does physical activity attenuate, or even eliminate, the detrimental association of sitting time with mortality? A harmonised metaanalysis of data from more than 1 million men and women. Lancet 2016;388:1302-10. 
27. Matthews CE, Chen KY, Freedson PS, et al. Amount of time spent in sedentary behaviors in the United States, 2003-2004. Am J Epidemiol 2008;167:875-81.

28. Gardner B, Smith L, Lorencatto F, et al. How to reduce sitting time? A review of behaviour change strategies used in sedentary behaviour reduction interventions among adults. Health Psychol Rev 2016:10:89-112.

29. Lally P, Gardner B. Promoting habit formation. Health Psychol Rev 2013;7:S137-S158.

30. Matei R, Thuné-Boyle I, Hamer M, et al. Acceptability of a theorybased sedentary behaviour reduction intervention for older adults ('On Your Feet to Earn Your Seat'). BMC Public Health 2015;15:606.

31. Sallis J, Owen N. Ecological models of health behaviour. Health behaviour and health education: theory, research, and practice. 3rd edn. San Francisco: CA: Jossey-Bass, 2002:462-484.

32. Merom D, Bauman A, Phongsavan P, et al. Can a motivational intervention overcome an unsupportive environment for walking--findings from the Step-by-Step Study. Ann Behav Med 2009;38:137-46.

33. Hall KS, McAuley E. Individual, social environmental and physica environmental barriers to achieving 10000 steps per day among older women. Health Educ Res 2010;25:478-88.

34. Moran M, Van Cauwenberg J, Hercky-Linnewiel R, et al. Understanding the relationships between the physical environment and physical activity in older adults: a systematic review of qualitative studies. Int J Behav Nutr Phys Act 2014:11:79.

35. Van Cauwenberg J, De Bourdeaudhuij I, De Meester F, et al. Relationship between the physical environment and physical activity in older adults: a systematic review. Health Place 2011;17:458-69.

36. Orstad SL, McDonough MH, Stapleton S, et al. A systematic review of agreement between perceived and objective neighbourhood environment measures and associations with physical activity outcomes. Env Behav (Published Online First: 29 Sept 2016)

37. Rosenberg DE, Kerr J, Sallis JF, et al. Promoting walking among older adults living in retirement communities. J Aging Phys Act 2012;20:379-94.

38. Baranowski T, Anderson C, Carmack C. Mediating variable framework in physical activity interventions. how are we doing? how might we do better? Am J Prev Med 1998;15:266-97.

39. Ryan RM, Deci EL. The Darker and Brighter sides of human existence: basic psychological needs as a unifying concept. Psychol Inq 2000;11:319-38.

40. van der Bij AK, Laurant MG, Wensing M. Effectiveness of physical activity interventions for older adults: a review. Am J Prev Med 2002;22:120-33.

41. Ng JY, Ntoumanis N, Thøgersen-Ntoumani C, et al. SelfDetermination Theory Applied to Health Contexts: a Meta-Analysis. Perspect Psychol Sci 2012;7:325-40.

42. Edmunds J, Ntoumanis N, Duda JL. Adherence and well-being in overweight and obese patients referred to an exercise on prescription scheme: a self-determination theory perspective. Psychol Sport Exerc 2007;8:722-40.

43. Williams GC, Niemiec CP, Patrick $\mathrm{H}$, et al. The importance of supporting autonomy and perceived competence in facilitating longterm tobacco abstinence. Ann Behav Med 2009;37:315-24.

44. Williams GC, McGregor HA, Zeldman A, et al. Testing a selfdetermination theory process model for promoting glycemic control through diabetes self-management. Health Psychol 2004;23:58-66.

45. Rouse PC, Ntoumanis N, Duda JL, et al. In the beginning: role of autonomy support on the motivation, mental health and intentions of participants entering an exercise referral scheme. Psychol Health 2011;26:729-49.

46. Thøgersen-Ntoumani C, Loughren EA, Taylor IM, et al. A step in the right direction? Change in mental well-being and self-reported work performance among physically inactive university employees during a walking intervention. Ment Health Phys Act 2014;7:89-94.

47. Thøgersen-Ntoumani C, Loughren EA, Duda JL, et al. "Step by Step". A feasibility study of a lunchtime walking intervention designed to increase walking, improve mental well-being and work performance in sedentary employees: Rationale and study design. BMC Public Health 2010;10:578.

48. Moore GF, Audrey S, Barker M, et al. Process evaluation of complex interventions: medical Research Council guidance. BMJ 2015;350:h1258.

49. Hox J multilevel analysis: techniques and applications. Mahwah, New Jersey: Lawrence Erlbaum Associates Publishers, 2002.

50. Chan AW, Tetzlaff JM, Altman DG, et al. SPIRIT 2013 statement: defining standard protocol items for clinical trials. Ann Intern Med 2013;158:200-7.
51. Hoffmann TC, Glasziou PP, Boutron I, et al. Better reporting of interventions: template for intervention description and replication (TIDieR) checklist and guide. BMJ 2014;348:g1687.

52. Australian Government Choose Health: be active [Internet]. Department of Health and Aging (DoHa). 2005. https://www.health gov.au/internet/main/publishing.nsf/Content/3244D38BBBEBD28 4CA257BF0001FA1A7/\$File/choosehealth-brochure.pdf. (citied 2016 Nov 15).

53. Vandelanotte C, Stanton R, Rebar AL, et al. Physical activity screening to recruit inactive randomized controlled trial participants: how much is too much? Trials 2015;16:446.

54. Cardinal BJ, Cardinal MK. Preparticipation physical activity screening within a racially diverse, older adult sample: comparison of the original and Revised Physical Activity Readiness Questionnaires. Res Q Exerc Sport 2000;71:302-7.

55. Kerr J, Rosenberg DE, Nathan A, et al. Applying the ecological model of behavior change to a physical activity trial in retirement communities: description of the study protocol. Contemp Clin Trials 2012;33:1180-8.

56. McClain JJ, Hart TL, Getz RS, et al. Convergent validity of 3 low cost motion sensors with the ActiGraph accelerometer. J Phys Act Health 2010;7:662-70

57. Clemes SA, Parker RA. Increasing our understanding of reactivity to pedometers in adults. Med Sci Sports Exerc 2009;41:674-80.

58. Washburn RA, Smith KW, Jette AM, et al. The Physical Activity Scale for the Elderly (PASE): development and evaluation. J Clin Epidemiol 1993;46:153-62.

59. Washburn RA, McAuley E, Katula J, et al. The physical activity scale for the elderly (PASE): evidence for validity. J Clin Epidemiol 1999;52:643-51.

60. Gardiner PA, Clark BK, Healy GN, et al. Measuring older adults' sedentary time: reliability, validity, and responsiveness. $\mathrm{Med} \mathrm{SCi}$ Sports Exerc 2011;43:2127-33.

61. Gandek B, Ware JE, Aaronson NK, et al. Cross-validation of item selection and scoring for the SF-12 health survey in nine countries: results from the IQOLA Project. J Clin Epidemiol 1998;51:1171-8.

62. Nelson $\mathrm{E}$, Wasson J, Kirk J, et al. Assessment of function in routine clinical practice: description of the COOP Chart method and preliminary findings. J Chronic Dis 1987;40 (Suppl 1):55S-63.

63. Lane D, Carroll D, Ring C, et al. Mortality and quality of life 12 months after myocardial infarction: effects of depression and anxiety. Psychosom Med 2001;63:221-30.

64. Zigmond AS, Snaith RP. The hospital anxiety and depression scale. Acta Psychiatr Scand 1983;67:361-70.

65. Bjelland I, Dahl AA, Haug TT, et al. The validity of the Hospital anxiety and depression Scale. an updated literature review. J Psychosom Res 2002;52:69-77.

66. Hughes ME, Waite LJ, Hawkley LC, et al. A short scale for measuring loneliness in large surveys. Res Aging 2004;26:655-72.

67. Bostic TJ, McGartland Rubio D, Hood M. A validation of the subjective vitality scale using structural equation modeling. Soc Indic Res 2000;52:313-24.

68. Zoellner J, Hill JL, Zynda K, et al. Environmental perceptions and objective walking trail audits inform a community-based participatory research walking intervention. Int J Behav Nutr Phys Act 2012;9:6.

69. Troped PCE, Fragala M, Melley S, et al. Development and reliability and validity testing of an audit tool for trail/path characteristics: the Path Environment Audit Tool (PEAT). J Phys Act Health 2006;5:746-60.

70. Williams GC, Grow VM, Freedman ZR, et al. Motivational predictors of weight loss and weight-loss maintenance. J Pers Soc Psychol 1996;70:115-26.

71. Ng JY, Ntoumanis N, Thøgersen-Ntoumani C. Autonomy support and control in weight management: what important others do and say matters. Br J Health Psychol 2014;19:540-52.

72. Niven AG, Markland D. Using self-determination theory to understand motivation for walking: Instrument development and model testing using Bayesian structural equation modelling. Psychol Sport Exerc 2016;23:90-100.

73. Braun V, Clarke V, Terry G. Thematic analysis. Qual Res Clin Health Psychol 2014;24:95-114.

74. Arain M, Campbell MJ, Cooper CL, et al. What is a pilot or feasibility study? A review of current practice and editorial policy. BMC Med Res Methodol 2010;10:67.

75. Teixeira PJ, Carraça EV, Markland D, et al. Exercise, physical activity, and self-determination theory: a systematic review. Int $J$ Behav Nutr Phys Act 2012;9:78.

76. Stathi A, McKenna J, Fox KR. Processes associated with participation and adherence to a 12-month exercise programme for adults aged 70 and older. J Health Psychol 2010;15:838-47. 